\title{
Lichen amyloidosis associated with chronic renal failure
}

\author{
Natsuko Matsumura, Toshiyuki Yamamoto
}

Department of Dermatology, Fukushima Medical University, Fukushima, Japan

Corresponding author: Dr. Natsuko Matsumura, E-mail: natsukom@fmu.ac.jp

Sir,

A 58-year-old Japanese male visited the Department of Dermatology of Fukushima Medical University Hospital, complaining of itchy eruptions on his trunk and lower legs. The skin rash had previously been treated with topical antihistamine cream, but without improvement. He had a past history of diabetic nephropathy and has been received peritoneal dialysis for 9 months. The patient's family history was noncontributory. Physical examination revealed a number of papulonodular eruptions on the trunk and hyperpigmented, keratotic, and firm papules and nodules on the lower parts of thighs and upper parts of shins (Fig. la and lb). Skin biopsy taken from the left leg showed epidermal hyperkeratosis and deposition of amorphous eosinophilic substance in the papillary dermis, which was evident on Congo-red and antcytokeratin antibody $34 \beta \mathrm{E} 12$ staining (Fig. 2a-c). Immunohistochemical staining of the $\beta 2$-microglobulin was negative. The clinical and histological findings confirmed lichen amyloidosis. We conducted half-side test by topical corticosteroid and moisturizing agent, in which the former was more effective. He achieved mild remission of symptoms in a period of one month.

Patients with chronic renal failure commonly exhibit cutaneous manifestations, such as pruritus, dry skin, hyperpigmentation, acquired perforating dermatosis, nephrogenic systemic fibrosis, calciphylaxis, porphyria cutanea tarda, and pseudoporphyria [1]. Furthermore, if dialysis is introduced, dialysis-related amyloidosis sometimes occurs during long-term course. In dialysisrelated amyloidosis, skin manifestations often present with cutaneous or subcutaneous nodules [2]. Bilateral subcutaneous masses are seen on the buttocks,

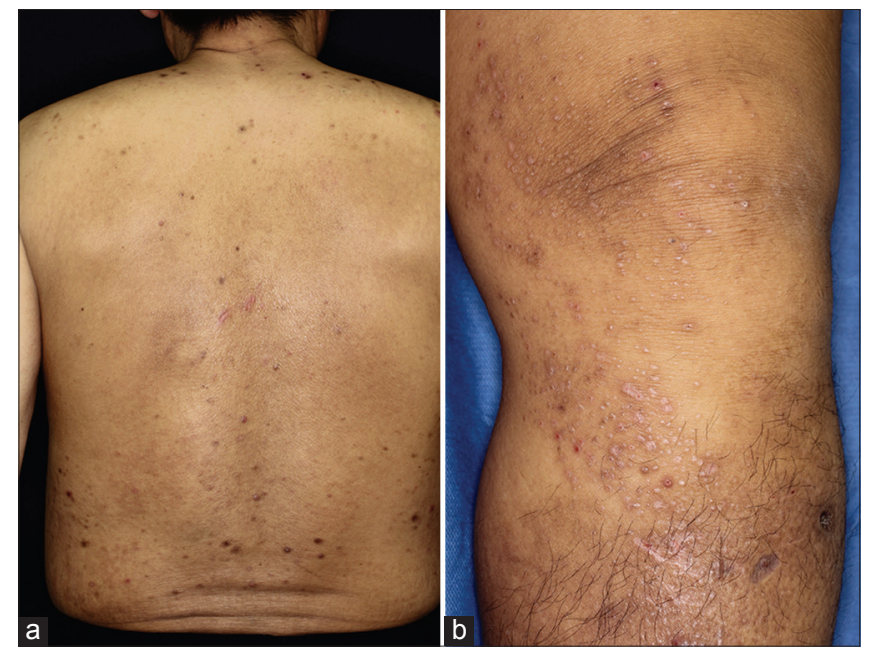

Figure 1: ( $a$ and b) Multiple, light brown papules appear and aggregate lower parts of thighs and upper parts of shins.

which are sometimes painful on sitting. Extensive deposition of $\beta 2$-microglobulin amyloid is seen in the dermis to subcutis, occasionally associated with local calcification. Beta2-microglobulin, which is not readily removed by hemodialysis deposits as amyloidosis. A previous report suggested that massive dermal and subcutaneous amyloid nodules on the buttocks of a patient on hemodialysis [3]. By contrast, in our case, we could not find nodular lesions on the buttocks.

Lichen amyloidosis frequently occurs on the extensor surfaces of the lower legs, and on the forearms and back. Lichen amyloidosis has been reported in associated with several skin disorders such as atopic dermatitis, lichen planus, C-type hepatitis, and mycosis fungoides [4,5]. However, lichen amyloidosis associated with chronic renal failure has not been reported, and to our knowledge, this is the first case of lichen amyloidosis in a patient with chronic renal failure. 


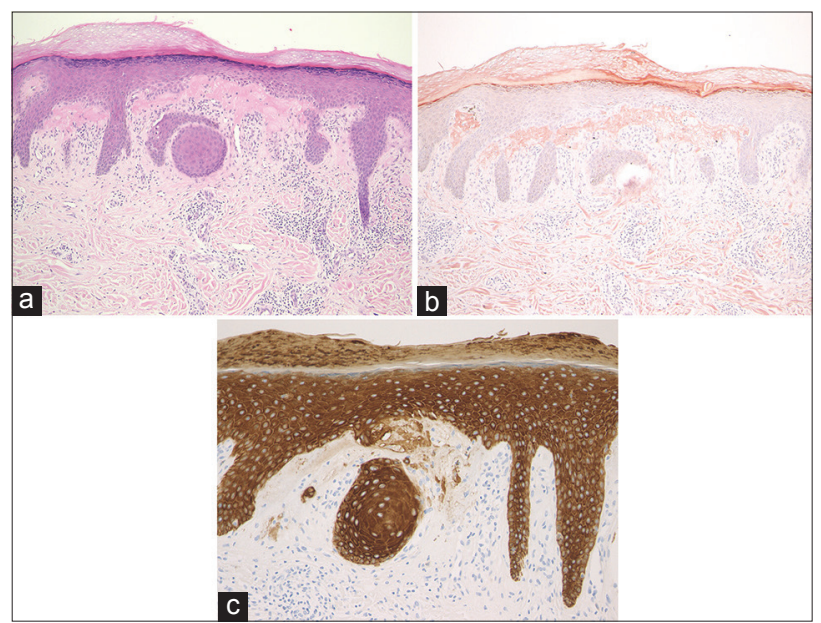

Figure 2: (a) Biopsy specimen showing dermal papillary deposits of eosinophilic materials (a), which were positively stained by Congo red (b) and ant-cytokeratin antibody $34 \beta \mathrm{E} 12$ (c). (original magnification; $a: \times 100, b: \times 100, c: \times 200)$.

The underlying cause of amyloid deposition is unknown. In our case, systemic amyloidosis was excluded by detail investigation. Several immunohistochemical studies have demonstrated reactivity between anti-keratin antibody and cutaneous amyloid deposits in lichen amyloidosis. Amyloid deposits may be derived from degenerated epithelial keratinocytes [6], possibly through filamentous degeneration or apoptosis [7]. In the present case, amyloid materials were also stained positive for $34 \beta \mathrm{E} 12$, suggesting that the origin of amyloid is degenerating epidermal keratinocytes which may represent precursors of cutaneous amyloid.
The patient complained of itching on the trunk and extremities, and he noticed frequent scratching. Thus, amyloid deposition on the lower legs may be induced by epidermal minor trauma induced by repetitive scratching.

\section{REFERENCES}

1. Robles-Mendez JC, Vazquez-Martinez O, Ocampo-Candiani J. Skin manifestations of chronic kidney disease. Actas Dermosifiliogr. 2015;106:609-2.

2. Takayama K, Satoh T, Maruyama R, Yokozeki H. Dialysis-related Amyloidosis on the Buttocks. Acta Derm Venereol. 2008;88:72-3.

3. Shimizu S, Yasui C, Yasukawa K, Nakamura H, Shimizu H, Tsuchiya K. Subcutaneous nodules on the buttocks as a manifestation of dialysis-related amyloidosis: a clinicopathological entity? $\mathrm{Br} \mathrm{J}$ Dermatol. 2003;149:400-4.

4. Behr FD, Levine N, Bangert, J. Lichen Amyloidosis associated with atopic dermatitis. Arch Dermatol. 2001;137:553-5.

5. Abe M, Kawakami Y, Oyama N, Nakamura-Wakatsuki T, Yamamoto T. A rare co-occurrence of primary localized cutaneous amyloidosis and chronic $\mathrm{C}$ type hepatitis. Int J Dermatol. 2010;49:960-9.

6. Chang YT, Liu HN, Wang WJ, Lee DD, Tsai SF. A study of cytokeratin profiles in localized cutaneous amyloids. Arch Dermatol. 2004;296:83-8.

7. Maeda H, Ohta S, Saito Y, Nameki H, Ishikawa H. Epidermal origin of the amyloid in localized cutaneous amyloidosis. Br J Dermatol. 1982;106:345-51.

Copyright by Natsuko Matsumura, et al. This is an open access article distributed under the terms of the Creative Commons Attribution License, which permits unrestricted use, distribution, and reproduction in any medium, provided the original author and source are credited.

Source of Support: Nil, Conflict of Interest: None declared. 